\title{
Un tema y tres variaciones: el caso Albert Nobbs
}

\author{
Aurora PIÑEIRO CARBALLEDA \\ Universidad Nacional Autónoma de México
}

A partir de la teoría de Linda Hutcheon sobre la adaptación, me propongo llevar a cabo una revisión de la novella "Albert Nobbs" (1918 y 1927), del escritor irlandés George Moore, como una fuente que fue adaptada para el ámbito teatral por Simone Benmussa (en 1977), y para el arte cinematográfico por Rodrigo García (en 2011). El análisis se centrará en las estrategias y variantes de caracterización relacionadas con el personaje central de la historia, y en cómo, a partir de ello, el tema de la identidad de género adquiere matices significativos que se tornan posibles gracias a los recursos de cada uno de los medios artísticos involucrados, la visión de los creadores que se apropian de la historia, y las distancias cronológicas, lingüísticas y culturales vinculadas con cada recreación.

PALABRAS ClaVE: adaptación, identidad de género, Albert Nobbs, George Moore, Simone Benmussa, Rodrigo García.

Bearing Linda Hutcheon's ideas on the theory of adaptation in mind, the aim of this article is to analyse "Albert Nobbs" (1918 and 1927), the novella by Irish writer George Moore, as a source that triggered Simone Benmusa's drama adaptation (in 1977) and Rodrigo García' film adaptation (in 2011). The analysis will focus on the characterisation strategies used by each artist to create the central figure of the story, and on how the theme of gender identity acquires distinctive traits which are made possible via the skillful use of conventions particular to each medium, as well as the principles of composition each one of the artists adopted in the process of recreating the story, taking into account the chronologic and lingüistical distances involved in every new rendering.

KEY WORDS: adaptation, gender identity, Albert Nobbs, George Moore, Simone Benmussa, Rodrigo García.

What trip would be more fascinating and exotic than to know what it truly is to be your own opposite? Or, indeed, to live in some elusive territory to which there is no opposite?

Andrew Solomon 


\section{Un primer acercamiento a la adaptación}

En el contexto artístico, los trasvases de un género literario a otro, o los ejercicios de intermedialidad que implican el "traslado" de una o más obras literarias a formas de representación como la escenificación, la adaptación cinematográfica, la ópera o el videojuego (para incluir creaciones de tipo interactivo) son objetos de estudio de la teoría de la adaptación y suscitan reflexiones diversas en torno a la naturaleza de cada uno de estos discursos, así como los distintos niveles de relación, dependencia o puesta en tela de juicio que caracterizan a cada producto que designamos bajo la categoría de una adaptación.

La teórica canadiense Linda Hutcheon se refiere a las adaptaciones como trabajos que emulan la práctica de la escritura en palimpsesto, es decir, como textos otros que guardan la huella de una escritura anterior. En este sentido, en el producto al que damos el nombre de adaptación conviven la obra(s) fuente con los elementos agregados o reformulados por el creador/a que identificamos como posterior, y el nuevo texto (en el sentido más amplio del término) se convierte en lo que Roland Barthes describía como una muy plural estereofonía de ecos, citas y referencias.

Para Hutcheon, la adaptación es "repetition, but repetition without replication. And there are manifestly many different possible intentions behind the act of adaptation: the urge to consume and erase the memory of the adapted text or to call it into question is as likely as the desire to pay tribute by copying" (2013: 7). En consonancia con lo anterior podemos agregar que abundan los ejemplos de adaptaciones en las que es posible identificar más de una intención a la vez, y el efecto, en apariencia contradictorio, entre homenaje y cuestionamiento de la obra que es punto de partida trae como resultado una tensión estética o de sentido, similar a la lógica de la figura retórica que conocemos con el nombre de paradoja. Hutcheon sintetiza su propuesta al señalar, a manera de lista, que la adaptación es:

-An acknowlegded transposition of a recognizable other work or works.

-A creative and an interpretive act of appropiation/salvaging.

-An extended intertextual engagement with the adapted work (8).

Por todo lo anterior, una adaptación es una derivación, pero no una obra derivativa, un trabajo que se puede llamar segundo, sin ser secundario. Al usar esta terminología, la estudiosa hace referencia al conocido trabajo de Gerard Genette, ${ }^{1}$ donde el subtítulo "literatura en segundo grado" no tiene la intención de ser despectivo con respecto a las distintas variantes de la reescritura que el teórico francés estudia. El texto adaptado es, entonces, para Hutcheon, su propia realidad palimpséstica, y desde los inicios de su análisis queda claro que no es pertinente juzgar la calidad del mismo desde un criterio de fidelidad, originalidad o antigüedad.

Con base en estas primeras consideraciones sobre la adaptación, me propongo llevar a cabo una revisión de la novella "Albert Nobbs" (1918 y 1927), del escritor irlandés George Moore, como una fuente que fue adaptada para el ámbito teatral por Simone

\footnotetext{
${ }^{1}$ Me refiero aquí a Palimpsests. Literature in the Second Degree. Channa Newman, Claude Doubinsky, trads.
} 
Benmussa (en 1977), y para el arte cinematográfico por Rodrigo García (en 2011). El análisis se centrará en las estrategias y variantes de caracterización relacionadas con el personaje central de la historia, y en cómo, a partir de ello, el tema de la identidad de género adquiere matices significativos que se tornan posibles gracias a los recursos de cada uno de los medios artísticos involucrados, la visión de los creadores que se apropian de la historia, y las distancias cronológicas, lingüísticas y culturales vinculadas con cada recreación.

\section{El caso Albert Nobbs}

El escritor irlandés George Augustus Moore (1852-1933), autor de una obra prolífica que incluye novela, cuento, ensayo, memorias, crítica de arte y una abultada correspondencia, dio vida al personaje de Albert Nobbs en una colección de cuentos publicada en 1918, con el título A Story-Teller's Holliday. En este libro el pretexto narrativo es un viaje de ida y vuelta entre Londres y el condado de Mayo, en Irlanda, durante el cual Alec Trusselby, un seanchai o contador de historias de la tradición gaélica irlandesa, y un tal George Moore, autor ficcionalizado, se turnan para contar historias. Cada texto está precedido por un diálogo entre George y Alec, y dicho intercambio introduce el relato que alguno de ellos contará. La historia de Albert Nobbs, narrada por George, es la de mayor extensión; de ahí que la crítica la considere una novella dentro de la colección de cuentos. La extensión es una de las razones por las que Moore decidió reeditar este texto dentro de otra colección de historias largas titulada Celibate Lives, que apareció en 1927. En este caso, el volumen cuenta con un prólogo que inicia a manera de ensayo, pero deviene en diálogo: una conversación ficcional entre Moore y Robert Louis Stevenson, que funciona como introducción a las cinco historias de vidas célibes, cada una con el nombre de su protagonista como título.

"Albert Nobbs", la tercera historia en la mencionada colección, es el relato de una vida en la que el celibato y la adopción de otra identidad de género se convierten en estrategias para sobrevivir en un mundo hostil donde la mujer, el bastardo o el desposeído, entre otros, son condenados a la ignominia o la muerte. El contexto es la Irlanda de mediados del siglo XIX, y Albert, una mujer que ha adoptado la identidad de varón, trabaja como mesero (casi mayordomo) en un afamado hotel de Dublín. Un día, el mayordomo es obligado a compartir habitación con un pintor de brocha gorda que ha laborado en el hotel y no tiene donde alojarse. El pintor, Hubert Page, descubre que Albert es mujer, y lo obliga a contarle su historia. Tras la narración desesperada de un Albert que implora no ser denunciado, Hubert confiesa que él también es mujer, y cuenta su historia, que incluye la huida de un matrimonio de juventud con un hombre violento; la posterior adopción de su identidad como varón, y el acuerdo matrimonial con otra mujer que funge como su esposa. La narración de la vida de Hubert sacude la estructura mental de Albert, y lo empuja a soñar con una existencia en la que él podría no estar solo. Así, concibe la idea de invertir sus ahorros en una propiedad, fundar una 
tabaquería, tener un hogar y una vida propios. Al día siguiente, despierta para descubrir que Hubert se ha marchado y que le hubiera gustado hacerle cientos de preguntas sobre cómo llevar una vida similar a la suya. En especial, sobre cómo conseguir una esposa, cómo y cuándo revelar a la candidata su verdadera identidad. La historia termina de manera trágica, con un Hubert viudo que regresa tiempo después, para enterarse de que Albert ha muerto, que su identidad sexual fue hecha pública de manera póstuma y que sus ahorros de toda una vida fueron confiscados por el gobierno. Hubert pasa la noche en la que había sido la habitación de Albert, tendido sobre la cama donde murió Albert, y sumido en una crisis que le lleva a considerar el regreso a su primera vida como un retorno posible, aunque el lector sospecha que semejante vuelta al pasado es un engaño, un acto de desesperación condenado al fracaso.

La historia de Albert Nobbs puede ser leída como una reformulación de las convenciones de la novela picaresca, donde el pícaro lucha por sobrevivir sirviendo a todo tipo de amos, carga con el estigma de una marca de nacimiento, busca llenar un vacío de identidad, e intenta ser aceptado en un grupo social que lo repele. Pero, a todo lo anterior, tenemos que agregar el ingrediente de la identidad de género. En términos sociales, Albert fue orillado a adoptar una identidad de varón, pero ha vivido tanto tiempo dentro del atuendo masculino que ya no cree posible volver a desempeñar el papel social de mujer: la ropa, y todo lo que ésta conlleva, ha tomado posesión de la persona, y Albert se define como "a perhapser": "neither man nor woman, just a perhapser" (Moore, 1927: 59), término que Moore acuña y utiliza para denunciar las limitaciones del lenguaje para nombrar la amplia variedad de la experiencia humana, otras posibles identidades de género que existen, se construyen y poseen una legitimidad propia, aunque no parezcan tener cabida en un mundo social y lingüísticamente constreñido.

No estamos frente a la adopción de la apariencia masculina (entendida aquí como el estereotipo más tradicional de lo masculino) como un simple disfraz temporal del que el personaje tendría la opción de desprenderse si las circunstancias así lo ameritaran, sino ante un ser humano que no puede volver a la identidad biológica y social con la que experimentó el mundo en un primer momento, y cuya historia, de manera gradual, provoca que el concepto de género se revele como una categoría que escapa al determinismo sexual y como una construcción mucho más compleja, en la que intervienen factores sociales y sicológicos múltiples. En el caso de Albert, el fatalismo se centra en la manera como llegó a esta elección: empujado por las circunstancias y con una fuerte dosis de dolor. De ahí la confusión y el desamparo que experimenta el personaje. La adopción de los ropajes masculinos es, en una primera instancia, un recurso de supervivencia: una armadura que lo protege de los asedios sexuales, así como de la explotación laboral de las mujeres al interior de un sistema patriarcal. Sin embargo, con el paso del tiempo, se da cuenta de que ha integrado un lenguaje corporal y una manera otra de estar en el mundo que se ha vuelto propia, y para la que no existe un sustantivo que materialice dicha condición, de ahí que el personaje se describa, en la cuarta y última parte del texto, como "only a shade" (84).

En el caso de Hubert Page, la obra nos entrega a una figura que, si bien construye su identidad masculina para escapar de circunstancias terribles, parece estar mucho 
más cómodo en su adopción de dicho rol, aunque la insinuada renuncia del final deja al lector con un triste sabor de boca. Los últimos párrafos de sus disquisiciones están caracterizados por una lacerante ambigüedad. Y la vuelta al diálogo entre los relatores iniciales como cierre del marco narrativo del texto incrementa el sentido de lo excepcional o la imposibilidad de que una historia como la de Hubert ocurra en un mundo diegético concebido en clave realista. La incorporación repentina de lo maravilloso está permeada, en el último párrafo de la novella, por lo condenatorio. Alec declara que Hubert tendría que explicar los quince años de ausencia de su primera vida matrimonial como producto del haber sido secuestrado/a por las hadas, y agrega: "A woman that marries another woman, and lives happily with her, isn't a natural woman; there must be something of the fairy in her" (96). Las líneas anteriores pueden abrir posibilidades menos ortodoxas para la interpretación de otros textos con personajes del mundo feérico pero, en el contexto de "Albert Nobbs", la lectura se inclina hacia un recordatorio de los principios con los que funciona el sistema social heteronormativo de la diégesis. El texto es subversivo en su exploración de temas tabú para la época, pero sus desenlaces, los completos y los parciales, permanecen en la órbita de lo trágico.

La dramaturga Simone Benmussa (1932-2001), nacida en Túnez y de nacionalidad francesa, fue una artista multifacética que lo mismo dirigía teatro, diseñaba escenografías o vestuario, que adaptaba obras de escritores reconocidos, muchos de ellos de la tradición en lengua inglesa. Su adaptación de la obra de Moore fue publicada y escenificada en francés, en 1977, con el título La vie singulière d'Albert Nobbs, y traducida al inglés como The Singular Life of Albert Nobbs, en 1979. ${ }^{2}$

La edición del texto dramático de Benmussa, publicada por Alma Classics, inicia con una introducción donde la autora rinde homenaje al respeto y ternura con que Moore trató a los personajes centrales de esta obra, pero también expresa su distanciamiento de la versión original, debido a las exigencias propias del cambio de medio, así como a su interés por subrayar ciertos aspectos de la historia o incorporar otros que obedecen a su visión personal, lo mismo que a las condiciones de escritura y producción relacionadas con las culturas europeas de finales de los años setenta: "one ransacks the text, one creates one's own reverie, one follows the subterranean layers of one's imagination and of one's own fantasies. One discovers different things" (2012: viii).

Los traslados de lo literario a las artes performativas son descritos por Linda Hutcheon como un movimiento del contar hacia el mostrar; en palabras de la teórica, "a move from the telling to the showing mode" (2013: 38). En el caso del teatro, nos encontramos con un medio caracterizado por una naturaleza doble: en primer lugar, la existencia del texto dramático como tal, cercano al ámbito literario por su condición de obra escrita aunque,

\footnotetext{
${ }^{2}$ Para este artículo se utilizó la traducción al inglés de Barbara Wright. La decisión de emplear el texto en su traducción al inglés busca que las tres fuentes primarias se encuentren en la misma lengua, además de ser el idioma en que la obra fue representada, durante muchos años, en la escena teatral internacional.
} 
al menos durante muchos años de la historia de la dramaturgia occidental, concebido para la escenificación. En segundo lugar, el fenómeno escénico, en el que intervienen factores diversos, entre los que se encuentran, como lo menciona José Luis Sánchez Noriega, el hecho de que la puesta en escena no oculte su carácter de representación, esté marcada por la irreproductibilidad de cada función, simplifique los soportes de la comunicación y funcione con la reducción al intercambio entre actores y espectadores (2000: 61).

En la transposición del texto de Moore a la obra de Benmussa encontramos una serie de diferencias formales, de las cuales mencionaré la estructura, ya que ésta me llevará de vuelta al tema que aquí nos ocupa. En el nivel de la estructura, la división de la novella en cuatro partes es sustituida por doce momentos o episodios de la historia, que tampoco corresponden a las partes o las escenas de la organización tradicional de un texto dramático. Esta disrupción de la convención dramática hace del ropaje formal de la obra un equivalente de los ropajes o atuendos transgresores de los personajes centrales, y dificulta la clasificación del corpus y de los cuerpos, tanto textuales como las presencias materiales en escena, bajo las etiquetas de uso corriente. La fluidez de la estructura y de las puestas en escena ${ }^{3}$ busca propiciar la añorada fluidez que el concepto de identidad de género encontrará tras la irrupción de los postestructuralismos en el ámbito intelectual de los años setenta en adelante. Benmussa acentúa la importancia, primero liberadora y después trágica, del vínculo entre la vestimenta/cuerpo y la configuración de una identidadde género al grado en que la adopción del atuendo masculino, en el caso de Albert, deja de ser una estrategia de supervivencia, para convertirse en una prisión y, por último, en una tumba. La idea del personaje que construye inadvertidamente su propia tumba se materializa en el énfasis que la dramaturga brinda $^{4}$ al gesto compulsivo de un Albert que sepulta billetes, amortajados en papeles de diversos colores, bajo las tablas del piso o en los recovecos de su habitación:

GEORGE'S MOORE VOICE: [...] She took to counting her money in her room at night. The half-crowns were folded up in brown-paper packets, the half-sovereigns in blue, the rare sovereigns were in pink paper, and all these little packets were hidden away in different corners... A sense of almost happiness awoke in her the day she discovered herself to be again as rich as she was before she met Helen. Richer by twenty-five pounds twelve and sixpence. Her eyes roved over the garret floor in search of a plank that might be lifted (Benmussa: 67-68).

\footnotetext{
${ }^{3}$ Me refiero, de manera específica, a la puesta en escena en el Théâtre d'Orsay, de París, en 1977, y a la puesta en el New End Theatre, en Londres, en 1978, ambas dirigidas por Benmussa, y con su propio diseño de escenografía.

${ }^{4}$ Por medio de la voz "en off" de un George Moore adulto que no tiene presencia en el escenario, pero que realiza intervenciones narrativas en tercera persona, que nos recuerdan que es él quien le está contando la historia a Alec, como en el texto original. Este elemento hibrida la obra, mantiene un rasgo narrativo al interior de un texto dramático. Además, la estrategia en cuestión establece un bello juego de inversiones especulares con la obra fuente: en el texto de Moore, los diálogos están asimilados al formato narrativo por la vía de la eliminación de guiones y comillas. En el de Benmussa, el discurso narrativo está incorporado al dramático, por la vía del formato de parlamento.
} 
El cuerpo se ve atrapado en una vestimenta, como los billetes envueltos y enterrados en una habitación que termina siendo proyección espacial del personaje, y su cámara mortuoria.

La puesta en escena también representa la dualidad y la percepción que Albert tiene de sí mismo como una sombra (a shade) al utilizar al personaje de una de las empleadas del hotel como una sombra que acompaña y reproduce los movimientos de Albert mientras éste fantasea con un futuro que nunca verá materializado. Una mujer se convierte en proyección inmaterial de otra, en un mundo que no ofrece ningún camino hacia la autonomía. Las páginas finales del texto vinculan, con un tono casi mordaz, los temas del determinismo social y las patologías con la siniestra referencia final de Alec a lo feérico, que ya habíamos comentado al analizar el texto de Moore. Sólo que estas líneas se ven acompañadas de una última imagen de Hubert Page, quien sueña, sentado sobre la cama donde murió Albert, mientras se escucha la voz de una mujer que canta, en irlandés, "Róisín dubh", o "Black Rose", una canción tradicional del siglo XVI, y que en Irlanda tiene claras connotaciones históricas de resistencia política. La utilización de varias canciones irlandesas en diversos momentos de la obra es una forma de representación de los distintos estados emocionales del personaje central, y esta última, "Black Rose", vincula a las dos figuras principales como protagonistas de una historia trágica de búsqueda de amor y titilantes aspiraciones de resistencia.

La obra de Benmussa demuestra que recursos como la estructura del texto, y lo sonoro o lo visual como elementos materiales de la puesta en escena, son estrategias filosas que potencian efectos específicos en la obra adaptada. Se trata de un ejercicio en el que el nuevo producto gana fuerza, ejerce un poder propio y resignifica la obra fuente. En el caso que acabamos de comentar, la creación de Benmussa se convierte, a su vez, en una de las fuentes utilizadas para la adaptación cinematográfica de la historia de Albert Nobbs.

En el año 2011, el cineasta colombiano Rodrigo García (1959) presentó su película (producida en inglés) Albert Nobbs: una adaptación tanto de la obra de Moore como de la de Benmussa, con un guión coescrito por John Banville, el narrador irlandés ganador del Man Booker Prize en 2005; Glenn Close, la actriz estadounidense que había representado el papel de Albert Nobbs en la escena teatral durante más de diez años; y la guionista húngara Gabriella Prekop.

Aunque el teatro y el cine comparten su calidad de artes performativas, el traslado del primer medio al segundo representa desafíos que han llevado a especialistas en el tema, como André Bazin o Sánchez Noriega, a llamar al teatro "el falso amigo" del cine (2000: 58). Sin embargo, en el caso de la adaptación realizada por García, los cambios en la estructura temporal de la obra fílmica fueron balanceados con una incorporación tersa de gran parte de los diálogos de los dos textos fuente, siendo éste un ejemplo de una lección de libertad para la adaptación bien aprendida de la obra de Benmussa.

El filme destaca por una cuidadosa recreación de época y un casting atinado. La actuación de Glenn Close como Albert fue celebrada por muchos críticos, aunque no faltaron las voces que la señalaron como demasiado elaborada. En el caso de Hubert Page, 


\section{$152 \square$ UN TEMA Y TRES VARIACIONES: EL CASO ALBERT NOBBS}

la interpretación de Janet Mcteer fue aclamada de manera unánime: la figura corpulenta y relajada del pintor de brocha gorda, así como el sesgo más claramente homosexual que el director brindó al personaje fueron bien recibidos por un público mucho más expuesto a la idea de la identidad de género como una construcción o una elección. A lo largo de la obra hay una alternancia de tomas panorámicas y close-ups que señala la tensión entre lo público y lo íntimo; y una serie de símbolos que representa la frontera entre estos dos ámbitos: las puertas, que se cierran en el mundo material, y las puertas que se abren en la esfera de la ensoñación; las cortinas y sábanas flotantes de la escena final, que develan y al mismo tiempo ocultan la posibilidad de otra existencia.

El tema de la identidad de género como construcción es abordado de manera más frontal en el filme. Una de las transformaciones importantes que García lleva a cabo en el tratamiento del mismo es la concreción material de varias escenas que en las fuentes anteriores sólo formaban parte de las fantasías de Albert Nobbs. Una de ellas es la visita al hogar de los Page: la posibilidad de observar la existencia de una relación de pareja distinta, y de que su historia sea escuchada por los dos personajes que representan una alternativa identitaria en la obra. Es en este contexto de solidaridad, y con un tempo narrativo más lento (si lo comparamos con el de la obra teatral), donde Albert expone su condición de bastardo/a, aborda el tema de la violencia sexual, e incluso, en una segunda visita (cuando Hubert ya es viudo), vive la experiencia de volver a portar ropajes femeninos. Se trata de una escena en la playa (García, Nobbs: 1:20), como espacio donde se tocan dos realidades: una líquida y cambiante, con otra sólida pero más maleable que la dura realidad urbana. La orilla del mar se convierte en el espacio limítrofe donde los personajes pueden jugar a vestirse de mujeres, para darse cuenta de que ya no saben cómo serlo, de que esa ropa es ahora el disfraz. Esta escena creada por García prescinde del diálogo: su narratividad se articula con la conjunción de recursos espaciales, visuales (que incluyen el trabajo corporal de los actores) y sonoros, es decir, con herramientas propias del lenguaje cinematográfico. A esta transformación se une la de un final diferente, incorporado por el cineasta como parte de una visión estética y política en la que el creador apuesta por un abordaje que brinda un respiro emocional a los personajes, al menos de manera temporal, antes de volver a la hostilidad del mundo.

En el caso de Hubert Page, de manera específica, se explora una homosexualidad que, si bien en sus inicios es la fuga de una realidad opresiva, se torna en elección. La obra fílmica reduce la cuota de ambigüedad respecto al tema de la homosexualidad femenina que se encontraba en estado latente en los textos anteriores: la materializa, y elige así una de las posibles lecturas insinuadas en las obras literarias.

\section{Algunas reflexiones finales}

En general, se piensa que la puesta en escena y la obra fílmica tienen mucho en común, debido a su carácter representacional y a que comparten el diálogo como forma discursiva. Sin embargo, la relación entre estos dos medios es muy compleja. Un ejemplo de 
ello es que, para el teatro, como lo explica Sánchez Noriega, el diálogo es consustancial a la concepción misma del texto dramático y el hecho escénico; mientras que, en la obra fílmica, el diálogo es uno más de los componentes de la comunicación audiovisual (2000: 61). El tiempo real y la condición de irrepetible del hecho escénico son muy distintos del tiempo y la inmutabilidad del filme concluido. Éstos y otros aspectos constituyentes de cada medio brindan al estudioso de la adaptación un campo fértil para la reflexión teórica y crítica sobre aspectos que van más allá de las coincidencias $o$ ausencias de acciones en el nivel de las tramas.

La revisión de las tres versiones de Albert Nobbs llevada a cabo en este artículo permite identificar la presencia de estrategias diversas de caracterización. En el caso del texto de Moore, el personaje central se construye a partir de los contrastes entre el punto de vista de otros personajes de la obra que lo describen y los parlamentos en los que Albert intenta describirse y enfrenta una crisis de vacío lingüístico para nombrar su condición. En el ejemplo de Benmussa, la caracterización se apoya en la estructura del texto, los juegos de proyección y duplicación corporal en escena, así como la incorporación de la música tradicional irlandesa para vincular la crisis identitaria individual con la del grupo social de las mujeres e, incluso, la identidad nacional periférica como otra forma de lo innombrable. Y, en un tercer momento, en la obra de García, el personaje central se asoma a variantes de la identidad de género que confirman la multiplicidad del concepto, aunque Albert no logre tener acceso pleno a las mismas. En todos los casos, la noción de identidad de género tradicional es desafiada por el texto, y la necesidad de pensarla desde otro lugar se hace patente.

Por último, la diversidad en cuanto a las nacionalidades, lenguas y medios de los artistas involucrados en las distintas adaptaciones de Albert Nobbs confirma la capacidad de dichos trabajos creativos para ejercer una doble relación de homenaje y distancia crítica con respecto a la obra fuente. Los textos adaptados conservan las huellas de la obra u obras anteriores, en coherencia con sus dinámicas en palimpsesto, pero las resignifican. Se convierten en trabajos de creación altamente intertextuales que refrendan el derecho a ejercer una apropiación del patrimonio artístico y recrearlo en un contexto que puede ser multicultural y de celebración, en más de un nivel, de la alteridad.

\section{Obras citadas}

Benmussa, Simone. 2012. The Singular Life of Albert Nobbs. Nueva York: Alma Books.

ButLer, Judith. 1990. “Gender Trouble, Feminist Theory, and Psychoanalitic Discourse”. Feminism/Postmodernism. Ed. Linda J. NichOLSON. Nueva York: Routledge. Pp. 324-340.

Cartmell, Deborah e Imelda Whelehan, eds. 2007. The Cambridge Companion to Literature on Screen. Cambridge: Cambridge University Press. 
Conway, Jill K., Susan C. Bourque y Joan W. Scott. 1997. "El concepto de género". El género: la construcción cultural de la diferencia sexual. Trad. Claudia LuCotti. Comp. Marta Lamas. México: unam, Pueg. Pp. 21-33.

DiAmOND, Elin. 1985. "Refusing the Romanticism of Identity: Narrative Interventions in Churchill, Benmussa, Duras". Theatre Journal, vol. 37, núm. 3. The Johns Hopkins University Press. Pp. 273-286. Web: 24 de enero de 2013. $<$ http://www.jstor.org/stable/3206848>.

Gaudreault, André y François Jost. 1995. El relato cinematográfico. Cine y narratología. Trad. Núria PuJoL. Barcelona: Paidós.

Genette, Gerard. 1997. Palimpsests. Literature in the Second Degree. Trads. Channa Newman y Claude Doublinsky. Lincoln / Londres: University of Nebraska Press.

HUTCHEON, Linda. 2013. A Theory of Adaptation. Nueva York / Londres: Routledge. JefFares, A. Norman. 2014. "Seriousness and Humour in the Novel". A Short History of Ireland's Writers. Dublín: The O'Brien Press. Pp. 62-74.

KeYssar, Helene. 1989. Reseña de The Feminist Spectator de Jill Dolan. Theatre Journal, vol. 41, núm. 3. John Hopkins University Press. Pp. 431-433. Web: 24 de enero de 2013. <http://www.jstor.org/stable/3208207>.

KIBERB, Declan. 2005. "George Moore's Gaelic Lawn Party". The Irish Writer and the World. Cambridge: Cambridge University Press. Pp. 91-104.

Moore, George. 1927. "Albert Nobbs". Celibate Lives. Londres: Heinemann. Pp. 44-96.

PeÑA-ARdid, Carmen. 1999. Literatura y cine. Madrid: Cátedra.

SABOURAUD, Frédéric. 2010. La adaptación. El cine necesita historias. Trad. Alica CAPEl TATJER. Madrid: Paidós.

SÁNCHEZ Noriega, José Luis. 2000. De la literatura al cine. Teoría y análisis de la adaptación. Barcelona: Paidós.

SANDERS, Julie. 2006. Adaptation and Appropriation. Nueva York / Londres: Routledge.

Solomon, Andrew. 2012. "Transgender". Far from the Tree. Parents, Children and the Search for Identity. Nueva York / Londres: Scribner. Pp. 599-676.

\section{Filmografia}

Albert Nobbs. 2011. Dir. Rodrigo GARCÍA. Reino Unido / Irlanda / Francia / Estados Unidos: Chrysalis Films / Canal+ / Irish Film Board / Mockingbird Pictures / Morrison Films / Parallel Film Productions / Trillian Productions / WestEnd Films. DVD. 